\section{The computation of retinal disparity}

\author{
ROBERT CORMACK \\ New Mexico Institute of Mining and Technology \\ Socorro, New Mexico \\ and

\section{ROBERT FOX} \\ Vanderbilt University, Nashville, Tennessee
}

One consequence of the horizontal separation of our eyes is that each eye receives slightly different patterns of stimulation. The images of objects nearer or farther than fixation fall on noncorresponding points on the retinas. These differences, which are referred to as retinal disparity, are responsible for the unique source of depth information known as stereopsis. For objects more distant than fixation, the images are displaced medially or nasally, and the disparity is called uncrossed or positive. Conversely, the images of objects nearer than fixation are displaced more laterally or temporally, and the disparity is called crossed or negative. The geometric relationships that produce the retinal disparity requisite for stereopsis are among the oldest and most thoroughly studied aspects of binocular vision. Helmholtz (1910/ 1962), Ogle (1962), Graham (1965), Gulick and Lawson (1976), and others have presented careful analyses of the geometry of retinal disparity.

Perhaps because the topic is old, treatments of the computation of disparity and of depth intervals for various kinds of viewing conditions are scattered and incomplete in much of the contemporary literature. A survey of 11 introductory textbooks on perception published since 1978 yields no specific formulas for the computation of retinal disparity. The works that do describe the geometry in detail either give no formulas convenient for computation or recommend simplified equations. These simplified approaches were extremely valuable before programmable calculators and microcomputers became widely available. But the approximations provided by the simplified formulas can be in error by significant proportions if their ranges of appropriate application are exceeded. One purpose of this note is to bring together, in a convenient way, information on methods of computation. A second purpose is to provide information on the amount of error generated by applying simplified formulas when their assumptions are violated.

We proceed by first examining the geometry that defines retinal disparity and related variables for the case

This work was supported in part by Grant EY00590-18 from the National Institutes of Health. Requests for reprints should be sent to either of the authors: R. H. Cormack, Department of Psychology, New Mexico Institute of Mining and Technology, Socorro, NM 87801; R. Fox, Department of Psychology, Vanderbilt University, Nashville, TN 37240. in which disparate objects are in 3-D space. Next, we derive the well-known simplifications of the correct formula and specify the assumptions underlying them. Finally, we note some of the consequences of applying these simplifications or approximations and the ranges within which errors may be tolerable.

\section{Explanation of the Computation of Retinal Disparity}

This computation assumes that all objects in depth are on the horizontal fixation plane or within a few degrees of it. The fixation plane is determined by the centers of rotation of the two eyes and the fixation point.

Referring to Figure 1: F is the fixated object. LE and $\mathrm{RE}$ are the left and right eyes, respectively. $\mathrm{M}$ is the midpoint between the centers of rotation $(1.3 \mathrm{~cm}$ behind the cornea) of the two eyes. I is the interocular distance, and $\mathrm{J}$ is half that value. ${ }^{1} \mathrm{Df}$ is the distance of the fixated object from the line connecting the centers of the two eyes. Af is the distance of the fixated object from the midsaggital plane, and is negative when fixation is to the left of the midsaggital plane.

Angle cf is the convergence angle and is the sum of angles 1 and 2 . The tangent of angle 1 is equal to the sum of $J$ and Af divided by Df. So:

$$
\text { angle } 1=\operatorname{ATN}[(\mathrm{J}+\mathrm{Af}) / \mathrm{Df}] \text {. }
$$

Remember that when fixation is to the left, Af is nega-

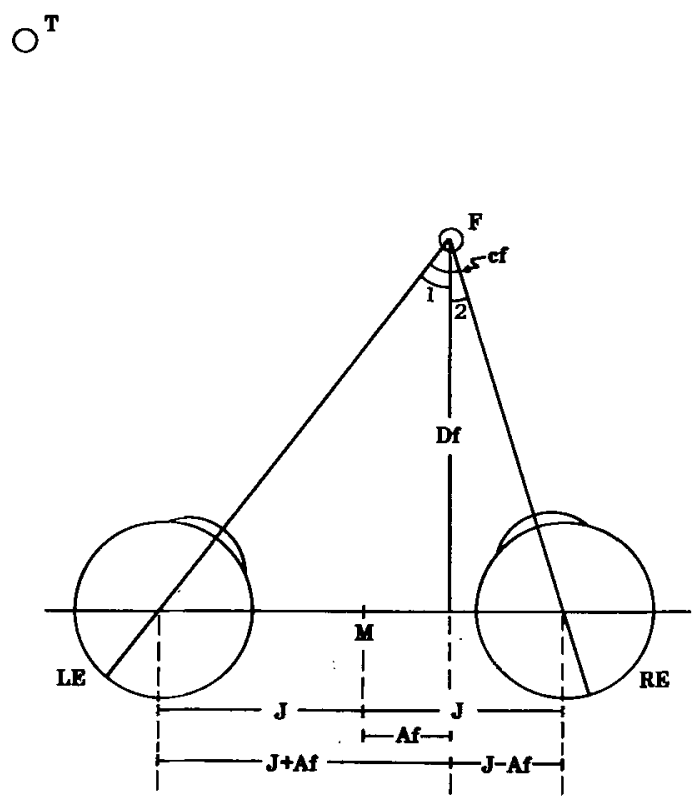

Figure 1. The geometric relationships that obtain when the eyes fixate a point, $F$, in space. The definition of lines and angles is given in the text. 
tive and $(\mathrm{J}+\mathrm{Af})$ is smaller than $\mathrm{J}$. By a similar procedure, angle 2 is:

$$
\text { angle } 2=\mathrm{ATN}[(\mathrm{J}-\mathrm{Af}) / \mathrm{Df}] \text {. }
$$

The convergence angle is:

$$
\text { angle } \mathrm{cf}=\text { angle } 1+\text { angle } 2 \text {. }
$$

Note that when the absolute value of Af is greater than half the interocular distance $(\mathrm{J})$, then, depending on whether fixation is to the right or left of the midsaggital plane, either angle 1 or angle 2 will be negative and convergence will be the difference between them.

Next, referring to Figure 2, we calculate the angle subtended by the eyes at the target in depth (i.e., the convergence angle ct at $\mathrm{T})$. This is equal to the sum of angles 3 and 4 . (Note that since angle 3 is negative, angle ct is smaller than angle 4.) This calculation is the same as that for angles 1 and 2 . Angle 3 is determined by:

$$
\begin{aligned}
& \text { angle } 3=\operatorname{ATN}[(J+A t) / D t] . \\
& \text { angle } 4=\operatorname{ATN}[(J-A t) / D t] .
\end{aligned}
$$

The sum of angles 3 and 4 gives angle ct:

$$
\text { angle } \mathrm{ct}=\text { angle } 3+\text { angle } 4 \text {. }
$$

Subtracting angle ct from angle cf gives the retinal disparity ( $r$ ) associated with target $T$ :

$$
\mathrm{r}=\text { angle } \mathrm{cf}-\text { angle } \mathrm{ct} \text {. }
$$

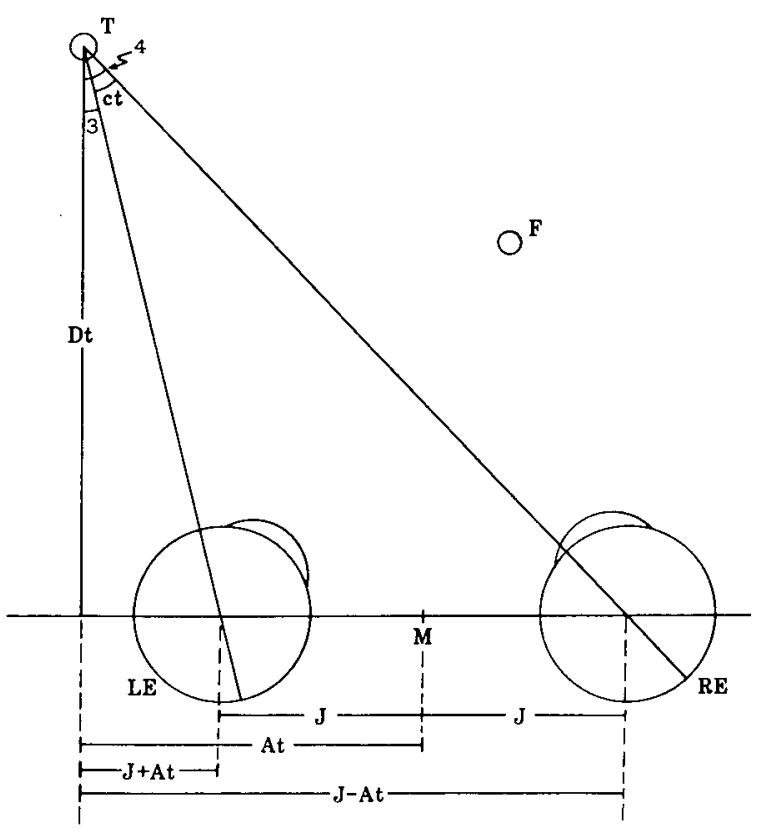

Figure 2. The geometric relationships that obtain when the eyes are stimulated by a tangent, $T$, in space while fixation is on $\mathbf{F}$.
So long as the eyes remain fixated on point $F$, angle cf will remain constant and the disparity of the other targets can be calculated from appropriate values of At and Dt.

Equations 1 through 7 are useful for expository purposes, but can be combined into the following equation:

$$
\begin{aligned}
\mathbf{r}=\{ & \operatorname{ATN}[(\mathrm{J}+\mathrm{Af}) / \mathrm{Df}]+\operatorname{ATN}[(\mathrm{J}-\mathrm{Af}) / \mathrm{Df}]\} \\
& -\{\operatorname{ATN}[(J+A t) / \mathrm{Dt}]-\operatorname{ATN}[(J-A t) / D t]\} .
\end{aligned}
$$

\section{Simplification in the Computation of Retinal Disparity}

One almost universal simplification made in the computation of retinal disparity is to assume that convergence is symmetrical and that targets in depth are on or near the midsaggital plane. This means that Af and At equal 0 , and removes them from Equations 1, 2, 4, 5, and 8 .

Removing Af and At from Equation 8 and simplifying leaves us with what we shall call the "trig law assuming symmetric convergence':

$$
r=[2 * \operatorname{ATN}(J / D f)]-[2 * A T N(J / D t)] .
$$

Since Dt is often expressed as fixation distance plus the depth interval (d), Equation 9 can be expressed as:

$$
\mathbf{r}=[2 * \operatorname{ATN}(\mathrm{J} / \mathrm{Df})]-\{2 * \operatorname{ATN}[\mathrm{J} /(\mathrm{Df}+\mathrm{d})]\} .
$$

Another very common simplification is to assume that, in radians, any angle equals its own tangent. For small angles, this is very nearly true, and applying it to Equation 10 and simplifying leads to the "nontrig law assuming symmetric fixation":

$$
\mathbf{r} \text { (in radians) }-\left(I^{*} \mathrm{~d}\right) /[\mathrm{Df} *(\mathrm{Df}+\mathrm{d})] .
$$

Finally, it is noted that when $D$ is large relative to $d$, that $D+d$ is roughly equal to $D$. This yields the very simple formula, often referred to as the distance-squared law:

$$
r \text { (in radians) }-\left(I^{*} d\right) / D^{2} .
$$

\section{Consequence of Simplifications}

We begin our discussion with the most extreme simplification and work up to approaches that yield more refined estimates. In each case, we have compared disparities calculated from Equation 8. For the most part, we present errors as percentages of the correct disparity. We have tried to note when the approximations are adequate, but since tolerable error depends upon the problem under study, researchers will have to use their own judgements in specific situations. We have selected $2 \%$ as a general tolerable error and give ranges of conditions under which $2 \%$ error will not be exceeded. For all calculations, we have assumed an interocular distance of $6.5 \mathrm{~cm}$.

Distance-squared law (Equation 12). In today's world of calculators and lab computers, there is no reason to 
use the so-called distance-squared law of retinal disparity (Equation 12). By assuming that fixation distance is equal to the fixation distance plus or minus the depth interval, one not only builds error into calculations, but also conceals the distinction between crossed and uncrossed disparity. It is important to note that the crossed disparity is greater than the uncrossed for any depth interval from any given fixation distance. This difference can be very large. At a fixation distance of $100 \mathrm{~cm}$, a depth of $+2 \mathrm{~cm}$ gives a true disparity of $4.38^{\prime}$, while a depth of $-2 \mathrm{~cm}$ gives a true disparity of $-4.56^{\prime}$ (a $4 \%$ difference). The distance-squared law gives $4.46^{\prime}$. As depth grows, the difference increases dramatically. A depth of $+10 \mathrm{~cm}$ gives a true disparity of $20.3^{\prime}$, and one of $-10 \mathrm{~cm}$ gives a true disparity of $-24.8^{\prime}$ ( $20 \%$ difference) contrasted with the $22.3^{\prime}$ approximation by the distance-squared rule. Depths of $+50 \mathrm{~cm}$ and $-50 \mathrm{~cm}$ give true disparities of $74^{\prime}$ and $-223^{\prime}$, respectively; the distance-squared law yields $111.1^{\prime}$. These last values are large depth intervals, but are encountered in research on depth constancy and related phenomena (see, e.g., Cormack, 1984). The distance-squared law gives the harmonic mean of the values for crossed and uncrossed disparities as computed by the nontrig law, assuming symmetric fixation, and as shown above is in error for both.

Nontrig law assuming symmetric fixation (Equation 11). So long as fixation is symmetric and the target in depth is on or within a few minutes of the midsaggital plane, this formula gives very accurate results within the range of usable disparities. For asymmetric fixation and fixation distances from $25 \mathrm{~cm}$ to infinity, errors in uncrossed disparity never exceed $2 \%$. For fixation distances from $50 \mathrm{~cm}$ to infinity, errors never exceed $2 \%$ within the range of $-2^{\prime \prime}$ to $-15^{\circ}$ disparity. For a fixation distance of $25 \mathrm{~cm}$ and crossed disparity, the error reaches $2 \%$ at a disparity of $-2.3^{\circ}$ and $5 \%$ at $-15^{\circ}$.

As with any formula that assumes symmetric fixation, the results from Equation 11 are in error whenever fixation or the target in depth is off the midsaggital plane. Since the errors are similar for Equations 9, 10, and 11, they are discussed only with reference to Equation 9 .

Trig law assuming symmetric fixation (Equation 9). This equation gives correct results so long as both fixation and the target in depth are on the midsaggital plane, so here we shall be concerned with horizontal assymmetries. The magnitude of the errors that result from ignoring asymmetry in the calculation of retinal disparity depends on fixation and target distances as well as on the size and sign of the disparity. The larger the disparity and the greater the fixation and target distances, the smaller is the effect of asymmetry. Asymmetry has greater effect for crossed than for uncrossed disparity, but the differences are not large except for large disparities $\left(>4^{\circ}\right)$. We will not attempt to discuss all possible combinations, but instead will concentrate on the condition most commonly encountered in research. This is where fixation is asymmetric (i.e., on the midsaggital plane) and the target is placed more laterally. This, by the way, is identical to the case in which fixation is asymmetric and the target is on the midsaggital plane.

Our calculations reveal that so long as the disparity is equal to or greater than $5^{\prime}$ (crossed or uncrossed), the error will be less than $2 \%$ for all fixation distances from $25 \mathrm{~cm}$ to infinity if the target is no farther than $1^{\circ}$ visual angle from the midsaggital plane. If the disparity is $1^{\circ}$ or more, then, for no more than a $2 \%$ error, the target should be within $2^{\circ}$ at $25 \mathrm{~cm}, 2.5^{\circ}$ at $50 \mathrm{~cm}$, and $3.5^{\circ}$ at $100 \mathrm{~cm}$. For disparities less than 15', these formulas should be avoided. For those who insist on using these simpler calculations, we can say that the error will be less than $2 \%$ if asymmetry of both the fixation point and the target is less than 10 times the disparity.

\section{General Conclusions Regarding Simplification}

Inasmuch as the proper calculation of disparity can be carried out on a computer or programmable calculator with a program of only a few steps, it would seem prudent to abandon the simplifications which were so valuable in earlier times.

\section{REFERENCES}

Cormack, R. H. (1984). Stereoscopic depth perception at far viewing distances. Perception \& Psychophysics, 35, 423-428.

ENRIGHT, J. T. (1984). Saccadic anomalies: Vergence induces large departures from ball-and-socket behavior. Vision Research, 24, 301-308.

GrahaM, C. H. (1965). Visual space perception. In C. H. Graham (Ed.), Vision and visual perception. New York: Wiley.

Gulick, W. L. \& Lawson, R. B. (1976). Human stereopsis: A psychophysical approach. New York: Oxford University Press.

HELMHOLTZ, H. voN (1962). Treatise on physiological optics (Vol. 3; J. P. C. Southall, Trans.). New York: Dover. (Original work published 1910)

OGLE, K. N. (1962). Spatial localization through binocular vision. In H. Davson (Ed.), The eye (Vol. 4). New York: Academic Press.

\section{NOTES}

1. There is some dispute regarding the appropriate measure of interocular distance. Since the nodal point (optical center) of the eye is not identical to the center of rotation, the optical center moves during vergence. Attempts to correct for this motion assume a rigid ball-and-socket rotation of the eyes. Enright's (1984) data call this assumption into question. Our own calculations (assuming rigid ball-and-socket action) show almost no effect of nodal point motions except under the most extreme conditions. Consequently, we are using the assumption that the nodal point and center of rotation are coincident.

To correct for nodal point motion under the assumption of ball-andsocket rotation requires a modification of the equations for computing angles 3 and 4 . The average distance of the nodal point from the center of rotation is $0.6 \mathrm{~cm}$ :

$$
\text { angle } 3=\mathrm{ATN}[(\mathrm{J}-\mathrm{X} 1+\mathrm{At}) /(\mathrm{Dt}-\mathrm{Y} 1)]
$$

where $\mathrm{X} 1=0.6 * \operatorname{SIN}($ angle 1$)$ and $\mathrm{Y} 1=0.6 * \operatorname{COS}$ (angle 1).

$$
\text { angle } 4=\operatorname{ATN}[(\mathrm{J}-\mathrm{Xr}-\mathrm{At}) /(\mathrm{Dt}-\mathrm{Yr})] \text {, }
$$

where $\mathrm{Xr}=0.6^{*} \operatorname{SIN}($ angle 2 ) and $\mathrm{Yr}=0.6 * \mathrm{COS}$ (angle 2).

(Manuscript received January 29, 1985; accepted for publication January 30,1985 .) 